\title{
Alvarado vs Lintula Scoring Systems in Acute Appendicitis
}

\author{
Daniel Ojuka, Mike Sangoro
}

School of Medicine, University of Nairobi

Correspondence to: Dr. Daniel Ojuka, P.O. Box 19762 - 00202, Nairobi, Kenya. Email: danielojuka@gmail.com

\begin{abstract}
Background: Timely and correct diagnosis of acute appendicitis reduces complications and avoids unnecessary surgeries. Scoring systems are used as adjuncts to aid in diagnosis. Methods: This was a prospective observational study carried out at Kenyatta National Hospital, from November 2014 to April 2015. Demographic, clinical and laboratory data was collected from consenting patients. The gold standard of diagnosis was histopathology. Data analysis was done using SPSS. The receiver operating characteristics, sensitivity, specificity, positive predictive values and negative predictive values of both scoring systems were then calculated for each of the scoring systems and compared between the two. Results: Majority of patients presented with right iliac fossa pain and underwent appendectomy.
\end{abstract}

\section{Introduction}

Acute appendicitis is a commonly diagnosed cause of the acute abdomen requiring emergency surgical intervention with an estimated lifetime prevalence of 1 in $7(1)$. However, making a correct and timely diagnosis remains a big challenge due to the occurrence of atypical signs and symptoms (2). This is especially true among females of child bearing age and older persons, the former in whom gynecologic conditions may mimic appendicitis (3). In order to aid in improving the accuracy of diagnosing acute appendicitis, scoring systems have been developed and put into regular clinical use. These include the Alvarado, Lintula, Fenyo-Lindberg, Appendicitis inflammation, Ohmann, Tzanakis and RIPASA scoring systems (4-10). Many of these scores utilize laboratory tests some of which may be difficult to assess quickly. Laboratory machines required may be out of reach or frequently break down, especially in resource limited settings. The two commonly used scoring systems are
The sensitivity of the Lintula and Alvarado scoring systems were $60.8 \%$ and $84.3 \%$ respectively, while the specificity was $60 \%$ and $35 \%$ respectively. The PPV of the Lintula was $79.5 \%$ while that of the Alvarado 76.8\%. Conclusion: The Lintula system compares well with the Alvarado system for diagnosis of acute appendicitis and can therefore be employed in situations where laboratory facilities may be limited.

Key words: Acute Appendicitis, Scoring systems, Lintula, Alvarado

Ann Afr Surg. 2017;14(1): 22-28.

DOI: http://dx.doi.org/10.4314/aas.v14i1.5

(C) 2017 Annals of African Surgery. This work is licensed under the Creative Commons Attribution 4.0 International License.

the Alvarado and the Lintula systems. While Alvarado scoring has clinical ( migrating abdominal pain, anorexia, nausea or vomiting, tenderness, rebound tenderness and elevated temperature) and laboratory( leukocytosis and shift to the left) components, the Lintula score is mainly clinical (gender, pain intensity, relocation of pain, location of pain, vomiting, elevated temperature, guarding, bowel sounds and rebound tenderness).The aim of this study was therefore, to compare the accuracy of two commonly applied scoring systems in the diagnosis of acute appendicitis, namely the Alvarado scoring system and the Lintula scoring system.

\section{Methods}

This was a prospective observational study that was conducted at the Kenyatta National Hospital (KNH) accident and emergency (A\&E) department and the surgical wards from November 2014-April 2015. The hospital is a national referral and teaching 
institution in Nairobi serving the entire country as well as neighboring countries. The study included all consenting patients above the age of 5 years who presented with abdominal pain suggestive of acute appendicitis. We excluded pregnant women, patients with features of generalized peritonitis, those who had undergone previous intra-abdominal surgery and those who were lost to follow up or had no means of communication.

Clinicians attending to these patients were continuously appraised and trained on the two scoring systems to be compared including the cut off points for diagnosis. This was done by one author or a trained research assistant who was a qualified clinical officer. A written card with the two systems was placed at the A\&E for ease of reference.

Concurrently, data sheets were provided for recording of the age and gender of the particular patient. Clinical data collected included abdominal pain character, intensity and whether it was migratory, vomiting, anorexia, nausea, temperature, tenderness, rebound tenderness, rigidity/guarding, bowelsounds, totalwhite blood cell counts and differentials and histopathology results for those who underwent surgery. Scoring was done using the data for each patient. Upon discharge a short-pretested questionnaire was used for follow up of their symptoms.

The decision on patient management; by discharge, observation, further diagnostic studies or appendectomy was left to the discretion of the treating clinician. The individual scores were then compared to the final diagnosis made by the clinician and to the recommendations of each scoring system. For those patients in whom the decision was made to undergo surgery, the final diagnosis of acute appendicitis was made by histopathology. Patients who were adjudged not to have appendicitis by the attending clinician had their telephone contacts noted for purposes of follow up and were discharged on analgesics. These patients were then contacted on day seven, to enquire about their symptoms. Specific details were sought on persistence, improvement or worsening of their symptoms, as well as information on whether they had sought medical or surgical intervention at any health facility for the same illness after discharge. Individuals who had resolution of their symptoms by the seventh day after discharge without having sought additional intervention were judged to have not had acute appendicitis. Using the data collected, the rates of negative appendectomy, sensitivity, specificity, positive predictive value (PPV) and negative predictive value (NPV) were calculated and values compared for each of the scoring systems.

Data was analyzed by use of SPSS version 22. Receiver Operating Characteristics (ROC) curve analysis was carried out. Additionally, sensitivity, specificity, PPV, and NPV were calculated. Differences between the sensitivity, specificity, PPV, NPV and overall accuracy rates were tested using the chi-square testing at 95\% confidence intervals. Pearson correlation co-efficient was used to determine the relationship between the Alvarado score, Lintula score and the histopathology result. Ethical approval was obtained from the ethics and research committee of the (KNH and University of Nairobi (UON)).

\section{Results}

This study was carried out on 71 patients aged between 5 years and 71 years with a mean age of $26.3( \pm 2.65)$ at the KNH A\&E department as well as within the surgical wards. While majority, 63(88.7\%) of the respondents' pain was located at the right iliac fossa, $4(5.6 \%)$ had generalized abdominal pain and $3(4.2 \%)$ had hypogastric pain. Only $1(1.4 \%)$ respondent reported pain in the epigastric region. The proportion of the respondents with pain in right iliac fossa was the highest as compared to patients with pain in other locations (Pairwise Binomial test $p<.001$ ). The difference in other locations was not significant. This implies that pain was most commonly located in the right iliac fossa. Pain had migrated from a different location for majority $45(63.4 \%)$ of the respondents while in $26(36.6 \%)$ pain had begun at the current location. The proportion of patients whose pain had migrated from a different location was significantly higher than the proportion of patients whose pain persisted at the same location (One Sample Binomial test $\mathrm{p}<0.001)$. Majority $(31 / 45)$ of the patients whose pain had migrated from a different location had pain migration from the periumbilical region.

Majority 61(85.9\%) of the respondents underwent appendectomy; 7(9.9\%) were discharged while $3(4.2 \%)$ underwent formal laparotomy due to what the treating clinicians described as peritonitis though no features of generalized peritonitis were described. Appendectomy was significantly the most prevalent decision taken by clinician as compared to both laparotomy and discharge (One sample Binomial test $\mathrm{p}<0.001$ ). Majority $26(42.6 \%)$ of the patients who underwent appendectomy had uncomplicated/simple 
acute appendicitis, 22(36.1\%) acute suppurative appendicitis, 3(4.9\%) gangrenous appendicitis while $10(16.4 \%)$ were found to have a normal appendix. All three patients who underwent laparotomy tested negative for appendicitis, with final diagnoses of TB peritonitis, peri-appendicitis and pyosalpinx. All the 7 patients who were discharged home reported resolutions of their symptoms with no intervention and were then considered negative for appendicitis. Thus, of all the patients recruited, a total of 20 (28.1\%) respondents had no acute appendicitis.

Male patients made up the majority 37(72.5\%) of those who had appendicitis on histopathology. Similarly, majority $11(55.0 \%)$ of patients who had negative appendectomy were male. There was no association between the respondent's gender and whether or not the respondents had appendicitis $(\mathrm{p}=0.155)$. Majority $46(90.2 \%)$ of patients who had appendicitis reported severe pain as did majority $16(80.0 \%)$ of patients who had negative appendectomy. There was no association between pain intensity and final diagnosis $(\mathrm{p}=0.245)$. It was also noted that majority $33(64.7 \%)$ of patients who had acute appendicitis had pain relocating from a distant site while a majority $12(60.0 \%)$ of patients who had no appendicitis were reported to have experienced similar relocation of their pain. There was no association between pain relocation and final diagnosis ( $p$-value $=0.711$ ). Evaluation of other Lintula scoring parameters is represented in Figure 1.

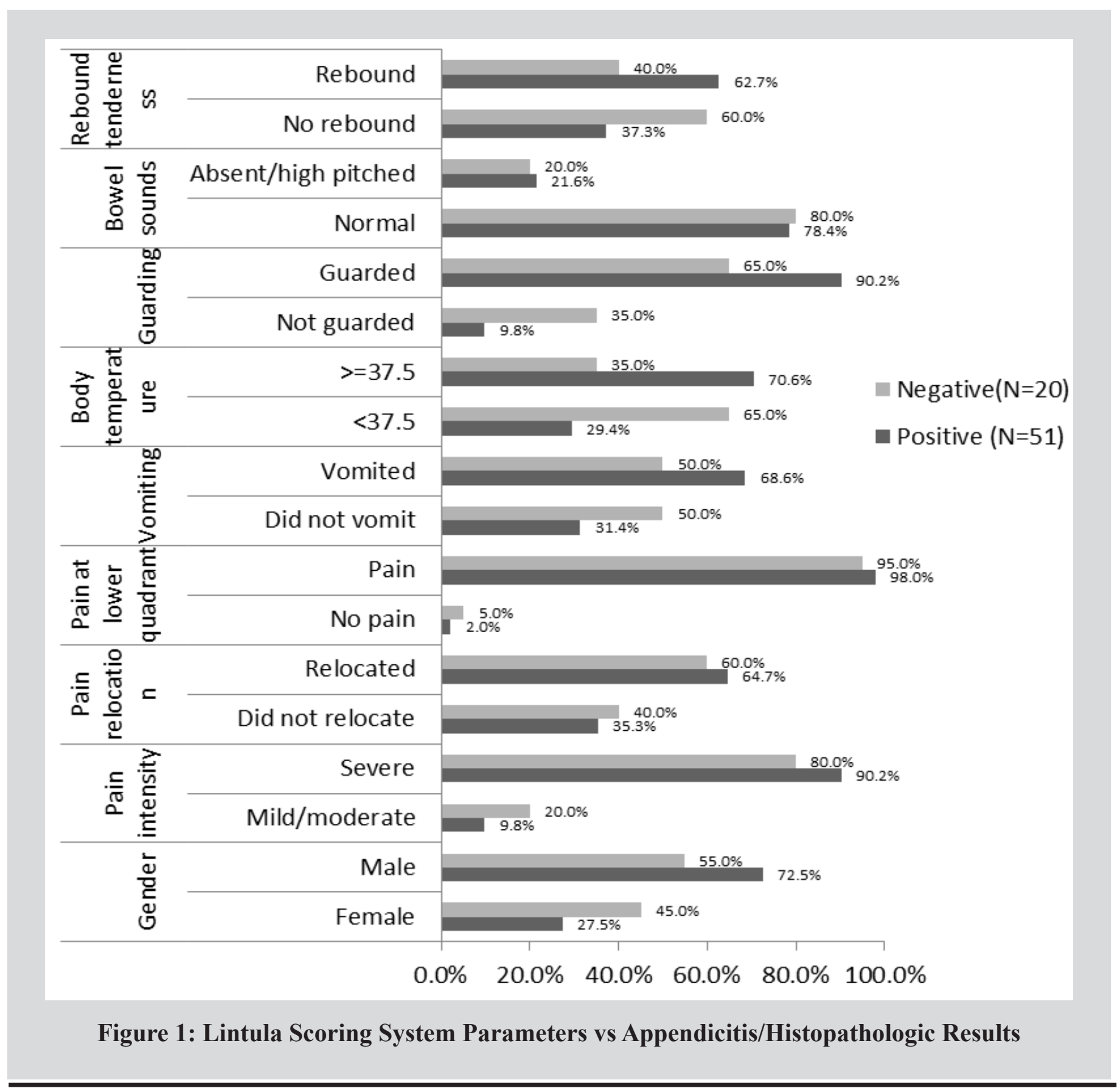

24 The ANNALS of AFRICAN SURGERY. January 2017 Volume 14 Issue 1 
Majority of the respondents (19.72\%) had a Lintula index of between 26 and 28 (Figure 2). Using the Alvarado system $31(60.8 \%)$ of patients who had appendicitis and $12(60.0 \%)$ of those who did not, had migratory pain. There was no association between migratory pain experience and final diagnosis ( $p$
$=0.951)$. Majority $47(92.2 \%)$ of patients who had appendicitis experienced anorexia as did majority $19(95.0 \%)$ of patients who did not have appendicitis. There was no association between experiencing anorexia and having acute appendicitis $(\mathrm{p}=0.674)$. Other results are presented in Figure 3.

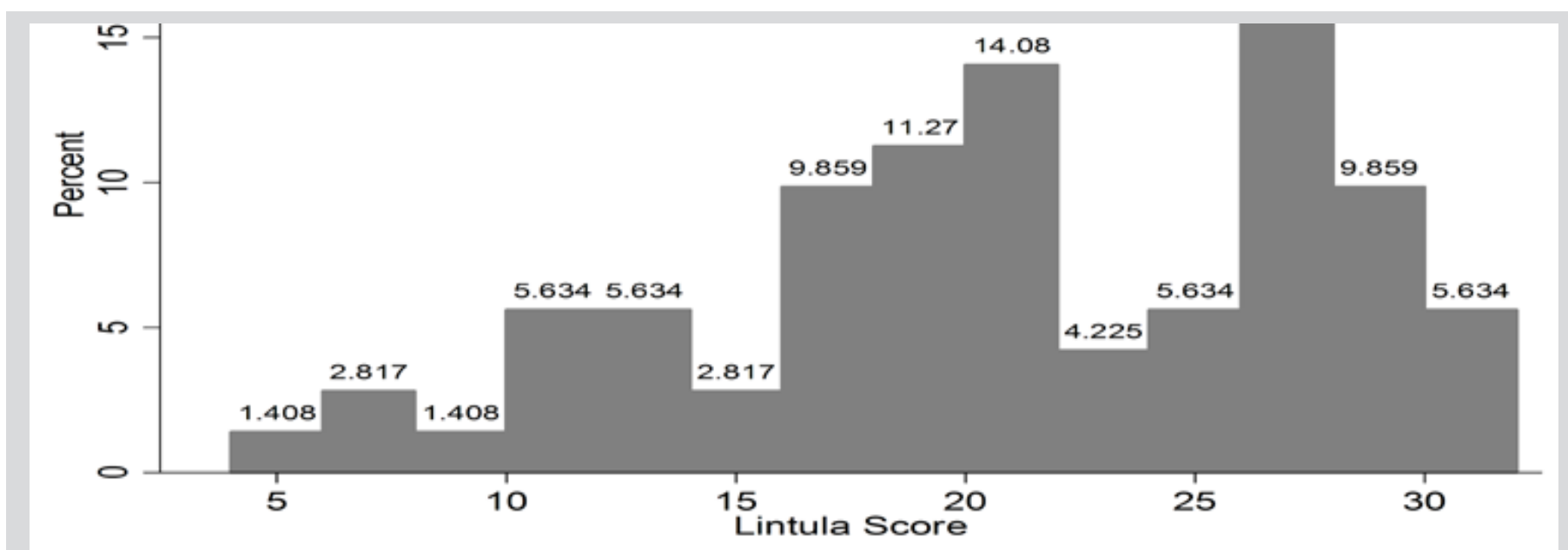

Figure 2: Distribution of Lintula Scores

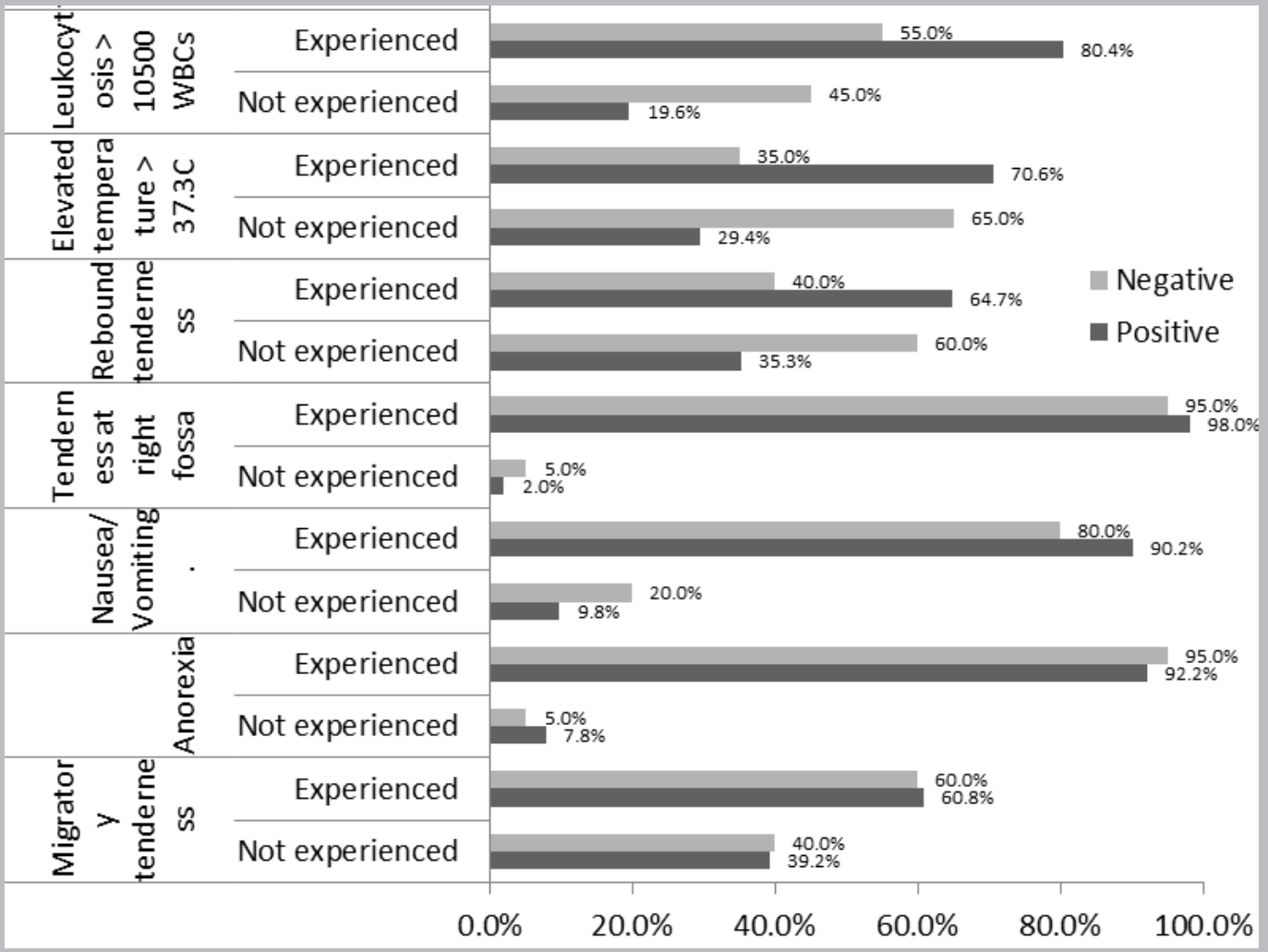

Figure 3: Alvarado Scoring System Parameters vs Appendicitis Results 


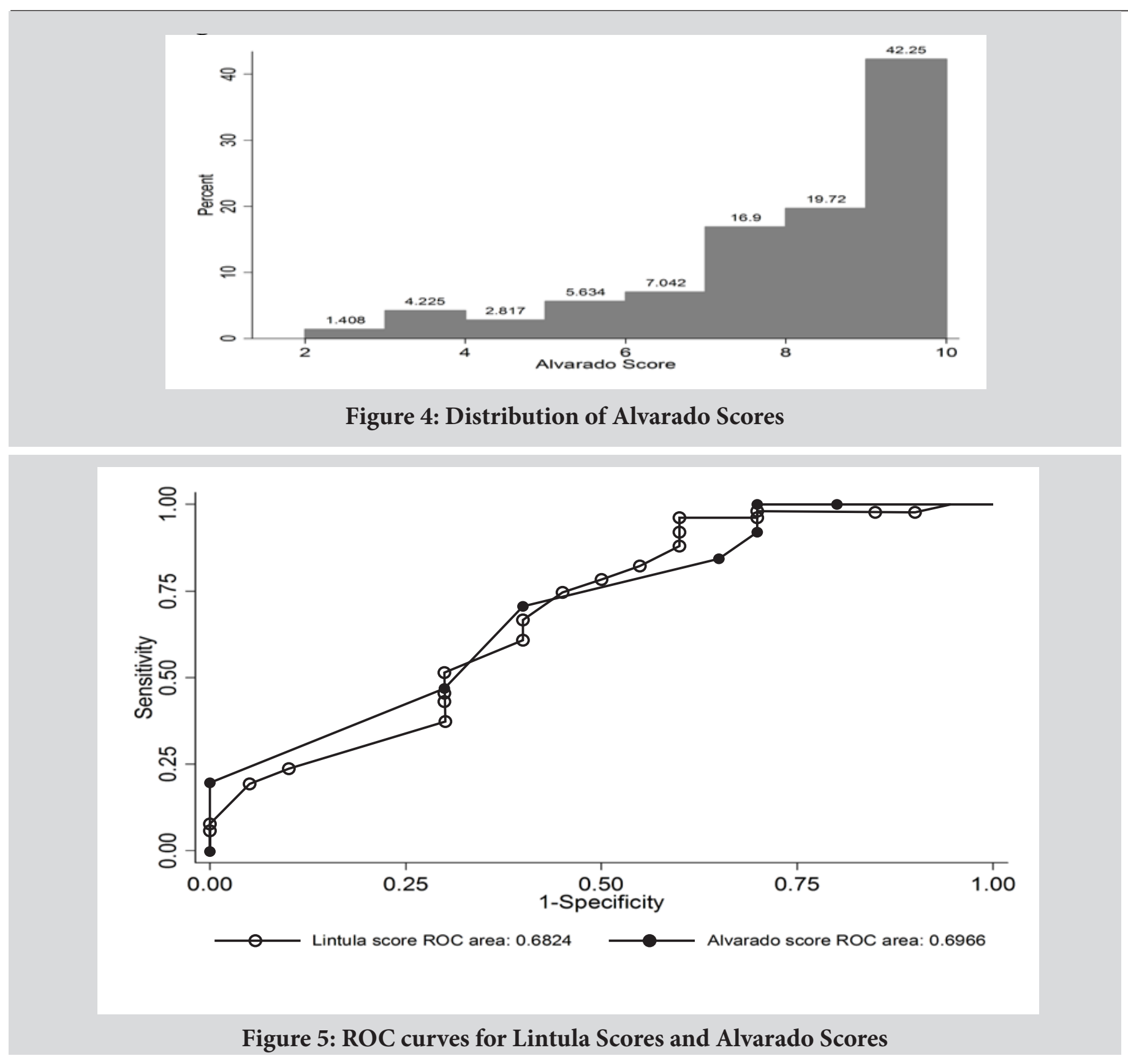

Table 1: Results at diagnostic cut-off points

\begin{tabular}{|l|l|l|l|l|}
\hline \multirow{2}{*}{ Appendicitis histology results } & \multicolumn{2}{|l|}{ Lintula test at cut off score of 21 } & Alvarado test at cut off score of 7 \\
\cline { 2 - 6 } & Negative & Positive & Negative & Positive \\
\hline Negative & 4 & 6 & 1 & 9 \\
\hline Positive & 20 & 31 & 8 & 43 \\
\hline
\end{tabular}

Majority (42.25\%) of the respondents had an Alvarado score index of between 9 and 10(Figure 4). There was a strong positive correlation between Lintula score and Alvarado score (Pearson Correlation Coefficient $=0.628, \mathrm{p}$-value 0.001$)$. A high value of Lintula score was associated with a high value of Alvarado score. The probability that Lintula score
(Area under Curve (AUC) $=0.6824, p=0.017$ ) will rank a randomly chosen positive instance higher than a randomly chosen negative one was higher than the probability that Alvarado score (AUC $=0.6966, \mathrm{p}=$ 0.010 ) will rank a randomly chosen positive instance higher than a randomly chosen negative one (Figure 5). There was no difference in the AUC in both scoring 
systems $(\mathrm{Z}$ - test $\mathrm{p}=0.8342)$. Of the 61 patients who underwent appendectomy, 51(83.6\%) had acute appendicitis on histopathology, while 10(16.4\%) did not have appendicitis. At a cut-off score of 21 for the Lintula scoring system, $6(9.8 \%)$ patients would have been falsely positive while at a cut off score of 7 for the Alvarado system 9(14.8\%) would have been falsely identified as having acute appendicitis (Table 1). There was no difference (Z-test $\mathrm{p}=0.407$ ) in the false positivity rate between the Lintula and Alvarado systems at these cut-off scores. At these points (21 and 7) the sensitivity of the Lintula system was $60.8 \%$ while the Alvarado system was $84.3 \%$. There was significant difference (z-test $p=0.008$ ) in sensitivity for both scoring systems at these points. At these points the specificity of Lintula system was $60.0 \%$ while Alvarado system was $35.0 \%$. There was no difference ( $z$-test $\mathrm{p}=0.114$ ) in specificity for both scoring systems at these points. At these points the positive predictive value (PPV) of the Lintula system was $79.5 \%$ compared to $76.8 \%$ for the Alvarado system. There was no difference (z-test $p=0.757$ ) in the PPV for both scoring systems at these points. The negative predictive value (NPV) of Lintula system was $37.5 \%$ while that of the Alvarado system was $46.7 \%$. There was no difference (z-test $p=0.549$ ) in the NPV for both scoring systems at these points. At these points the overall accuracy of Lintula system was $60.6 \%$ while Alvarado system was $70.4 \%$. There was no difference (z-test $\mathrm{p}=0.215$ ) in overall accuracy for both scoring systems at these points. From the ROC curve analysis, the AUC, sensitivity, specificity, PPV and NPV values for both scoring systems were similar. This implies that at the optimal cut-off scores, neither the Lintula nor Alvarado Scoring systems was superior to the other in diagnosing or ruling out acute appendicitis.

\section{Discussion}

Various scoring systems have been developed around the world in an attempt to objectively assist clinicians make an accurate diagnosis as well as reliably rule out acute appendicitis (4-10). While many clinicians employ the Alvarado system routinely, there has been need to identify other more accurate, simpler and more cost-effective scoring systems for diagnosing or ruling out acute appendicitis. The Lintula scoring system was originally developed for the pediatric age group, but has since been validated for use in the adult age group (5).

The mean age of this study varies with a retrospective study by Konan et al which compared the two systems in which the patients whose records were reviewed had a median age of 69 years (range 65-83 years) (11). It also differs with a prospective study by Sencan et al involving 60 pediatric patients with a mean age of 9.9 years (12). Kirkil et al have also conducted a comparative study though retrospective, using 114 patients' records but in which no age or gender characteristics are described (13).

In our study, we found a significant difference in the sensitivity of the Lintula and Alvarado scoring systems, but no significant differences in specificity, PPV, NPV and overall accuracy between both scoring systems for the diagnosis of acute appendicitis. Kirkil et al. compared the accuracies of the Lintula and Alvarado scoring systems and reported sensitivity of $83.9 \%$ and 95.5\% for the Lintula and the Alvarado respectively $(p=0.007)$ (13). Though reaching a similar conclusion to our study as regards the difference in sensitivities, these values are high compared to our findings, which may have been attributed to the larger sample size in that study. The specificity in the same study was $96.4 \%$ and $67.6 \%$ for the Lintula and the Alvarado systems respectively $(p=0.001)$. This varies with our study which reports no difference in specificities between the two systems. The PPV, NPV and overall accuracy was comparable between the two scoring systems. The negative appendectomy rate was $17.2 \%$ and they estimated that had the Alvarado or Lintula systems been exclusively used in diagnosing their patients, this rate would have been $18.5 \%$ and $3.6 \%$ respectively. This compares with our study in which the negative appendectomy rate was $16.4 \%$ and would have been $14.8 \%$ and $9.8 \%$ had the Alvarado or Lintula systems been exclusively used, respectively. It is worth noting that in the study, comparison of the accuracies of the two systems was a secondary objective and no age or gender sub analysis was carried out (13).

Konan et al, in their comparison of the two systems in the geriatric age group found the accuracies to be $85 \%$ and $78.7 \%$ for Alvarado and Lintula respectively at cut-off scores of 7 and 21(11). The variance with our study may be attributable to the age group in which the study was conducted. No comparison was made on the rate of negative appendectomy between the two systems in that study. 
Sencan et al found that the two systems had no predictive value as well as insufficient sensitivity and specificity and thus had no advantage over clinical acumen and experience (12). Our results imply that the Alvarado scoring system, the most commonly applied system in our setup, may have similar negative appendectomy rates with the Lintula scoring system. Our study also found no significant association between the patient's gender and whether or not they were likely to have acute appendicitis.

Because of the similarities, the Lintula scoring system has the inherent advantage in that it does not require laboratory parameters. This then means that it can be deployed for use in situations where laboratory facilities may be difficult to access. The diagnosis of acute appendicitis, whose gold standard remains histopathology, remains a challenging endeavor even with the deployment of diagnostic adjuncts such as ultrasound and CT scan. In this study, it was noted that all patients with a presumptive diagnosis of acute appendicitis had routine ultrasonography. This was despite the recommendations that diagnostic adjuncts such as imaging be employed at equivocal score cutoffs of 16-21 for Lintula and 5-6 for the Alvarado system $(4,5)$. Despite the deployment of ultrasonography as well as the clinician's acumen, a negative appendectomy rate of $16.4 \%$ was observed. However, in our estimation, had the Alvarado scoring system been exclusively applied to all patients, the rate would have been approximately $14.8 \%$ compared to $9.8 \%$ for the Lintula scoring system.

The limitation of this study is in the low numbers accrued during the period of study.

\section{Conclusion}

This study demonstrates that there is no difference in the overall diagnostic accuracy of the Lintula system compared to the Alvarado system for the diagnosis of acute appendicitis. The Lintula scoring system is a simple, non- invasive and cost effective way of narrowing down the diagnosis of acute appendicitis with potential utility in resource limited settings. We recommend however, that in the application of the Lintula system, it should be borne in mind that its sensitivity may be lower than the Alvarado system. Further studies may however be needed to compare the diagnostic accuracy in different age groups as well as between the genders.

\section{References}

1. StephensPL,MazzuccoJJ.ComparisonofUltrasound and the Alvarado Score for the Diagnosis of Acute Appendicitis. Conn Med. 1999;63: 137-40.

2. Khairy G. Acute Appendicitis: Is Removal of Normal Appendix Still Existing and Can We Reduce its Rate? Saudi J Gastroenterol. 2009; 15(3): 167-70.

3. Hatipoglu S, Hatipoglu F, Abdullayev R. Acute Right Lower Abdominal Pain in Women of Reproductive Age: Clinical Clues. World J Gastroenterol. 2014; 20(14):4043-9.

4. Alvarado A. A Practical Score for the Early Diagnosis of Acute Appendicitis. Ann Emerg Med. 986; 15:557-64

5. Lintula H, Kokki H, Pulkkinen J, et al. Validation of a Diagnostic Score (Lintula Score) for Adults with Suspected Appendicitis. Langenbecks Arch Surg. 2010; 395(5):495-500.

6. Fenyo G, Lindberg G, Blind $P$ et al. Diagnostic Decision Support in Suspected Acute Appendicitis: Validation of a Simplified Scoring System. Eur J Surg. 1997; 163(11):831-8.

7. Andersson M, Andersson RE. The Appendicitis Inflammatory Response Score: A Tool that Outperforms the Alvarado Score. World J Surg2008; 32(8):1843-9.

8. Ohmann C, Yang Q, Franke C. Diagnostic Scores for Acute Appendicitis. Abdominal Pain Study Group. Eur J Surg. 1995; 161(4): 273-81.

9. Tzanakis NE, Stamatis P, Danulidis K, et al. A New Approach to Accurate Diagnosis of Acute Appendicitis. World J Surg. 2005; 29(9):1151-6.

10. Chong CF, Adi M, Thien A, et al. Development of the RIPASA Score: A New Appendicitis Scoring System for the Diagnosis of Acute Appendicitis. Sing Med J. 2010; 5(3):220-5.

11. Konan A, Hayran M, Kilic YA, et al. Scoring Systems in the Diagnosis of Acute Appendicitis in the Elderly. Turkish J of Trauma and Emerg Surg. 2011;17(5):396-400.

12. Sencan A, Aksoy N, Yildiz M et al. Evaluation of Validity of Alvarado, Eskelinen, Lintula and Ohmann Scoring Systems in Diagnosing Acute Appendicitis in Children. Paediatr Surg Int 2014; 30(3):317-21.

13. Kirkil C, Karabulut K, Aygen E, et al. Appendicitis Scores may be Useful in Reducing the Costs of Treatment for Right Lower Quadrant Pain. Turkish J Trauma and Emerg Med. 2013;19(1):13-1. 